This journal is the official publication of Bangladesh Society of Physiologists (BSP)

Web URL: www.banglajol.info/index.php/JBSP

Abstracted /indexed in Index Copernicus, Director of Open Access Journal, HINARI Index Medicus for South East Asia Region, Google Scholar, 12OR, infobse index, Open J gate, Cite factor, Scientific indexing services

pISSN-1983-1213; e-ISSN-2219-7508

Article

Article information:

Received: 28th January 2020

Accepted: 29th May 2020

DOI: https://doi.org/10.3329/jbsp.v15i1.48113

Corresponding author:

Bithi Mallik, Department of Physiology,

Bangabandhu Sheikh Mujib Medical University,

Shahbag, Dhaka.

Email: bithimallik1985@gmail.com

Cite this article:

Mallik B, Ferdousi S, Sultana S. Effect of music therapy on heart rate variability in generalized anxiety disorder: A poincaré analysis

J Bangladesh Soc Physiol 2020;15(1): 39-45

This article is open access licensed under CC BY NC SA which allows readers copy, distribute, display, and perform the work and make derivative works based on it only for noncommercial purposes.

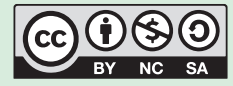

Volume 15 No. 1 June 2020: 39-45

\section{Effect of music therapy on heart rate variability in generalized anxiety disorder: A poincaré analysis}

\author{
Bithi Mallik $^{1}$, Sultana Ferdousi ${ }^{1}$, Shamima Sultana ${ }^{1}$ \\ 1. Department of Physiology, Bangabandhu Sheikh Mujib Medical University, \\ Shahbag, Dhaka.
}

\section{Abstract}

Background: Poincare plot analysis of heart rate variability (HRV) is a complex nonlinear method for assessing cardiac autonomic nerve function (CANF) in generalized anxiety disorder (GAD). Objective: This study aimed to observe the effect of music therapy on HRV by poincaré plot analysis in GAD patients. Methods: This prospective Interventional study was done in 2019 on 60 newly diagnosed GAD patients aged 20- 40 years, both male and female. Among them, 30 patients were under music therapy with Raga Bhairabi based Rabindra Sangeet for 3 months and 30 patients were without music therapy and both patient groups were studied over three months. Healthy controls, who were enrolled in this study, were Age, sex and BMI matched. Poincaré plot (non linear measure) of HRV of all GAD patients and control were recorded at baseline and after three months of follow up. HRV were recorded by Power Lab 8/ 35. For statistical analysis Bonferroni's Post Hoc test and paired sample ' $t$ ' test were done. Results:Standard deviation of the points perpendicular to the axis of line of identity(SD1), standard deviation of the points along the axis of line of identity (SD2) $\mathrm{SD}$ and SD1/SD2 were significantly lower in all GAD patients compared to healthy control at baseline. After 3 months of music therapy, significant increment of these parameters occurred in GAD patients, whereas no changes of these except SD1 were noted in patients without music therapy after 3 months. Moreover, these parameters were not significantly different in patients under music therapy compared to control at the end of three months. Conclusion: This study concluded that music therapy can improve cardiac autonomic dysfunction in GAD patients.

Keywords: Music, SD, SD2, GAD. 
Introduction

$\mathbf{G}$ eneralized anxiety disorder (GAD) is a common psychiatric disorder among people of all ages across the world affecting $3.1 \%$ of global population. ${ }^{1}$ The prevalence of GAD is $1.5 \%-3 \%$ in developing countries $^{2}$ and 6 million people are suffering from GAD in Bangladesh. ${ }^{3}$ GAD patients' often experience chronic and severe uncontrolled anxiety for at least 6 months, which is beyond control and associated with restlessness, lack of concentration, irritability, muscle tension, fatigue and sleep disturbance. ${ }^{1}$

Previous studies reported a significant association between GAD and autonomic dysfunction due to presence of autonomic symptoms. Autonomic dysfunction is characterized by overactive sympathetic drive and hypoactive parasympathetic function. ${ }^{4-7}$

Heart Rate Variability (HRV) is most widely used tool for detecting autonomic dysfunction by providing a quantitative assessment of individual component of cardiac autonomic tonic activity. It represents the variation of consecutive heart beat interval as well as instantaneous heart rate resulting from central and peripheral autonomic modulation. It can measure the output from central autonomic modulation. ${ }^{8}$ Nonlinear method of measurement of HRV represents a potentially effective tool for HRV assessment and among all nonlinear methods, poincaré plot is the most common method, used by the commercial devices for this purpose. Here, all the heart beats are geometrically represented and it helps the visual identification of the presence of non linear HRV. The Poincare plot is quantified by measuring SD1, SD2 and SD1/SD2 components where SD1 measures short term variability representing parasympathetic tone and SD2 measures long term HRV indicating both sympathetic and parasympathetic status. SD1/ $\mathrm{SD} 2$ reflects the proportion of short term and long term variability and evaluates sympathovagal balance. ${ }^{9-12}$
GAD has been shown to influence autonomic modulation. Several previous studies strongly suggest autonomic dysfunction is associated with GAD. ${ }^{4-7}$ Recent research evidence on the benefit of music therapy (MT) has been the basis of its use in various clinical conditions. Research evidence demonstrated therapeutic effect of music in preoperative and postoperative patients, cancer patients, autism patient, metabolic syndrome, schizophrenia, major depressive disorder and many other conditions, ${ }^{13-16}$ but very few studies investigated the effects of music therapy on GAD. With other standard care, music also has a beneficial role. In clinical settings, GAD is usually treated by medication together with psychiatric counseling in combination. A randomized control trial demonstrated the value of music therapy to reduce anxiety levels in $\mathrm{GAD}^{18}$. But the potentials of music therapy to improve autonomic dysfunction in GAD has not yet been explored. Therefore, this study has been designed to investigate the effect of music therapy on cardiac autonomic nerve function, analyzing by Poincaré plot of HRV in GAD patients.

\section{Methods}

This prospective interventional study was carried out from March 2019 to February 2020. Sixty (60) newly diagnosed $\mathrm{GAD}^{1}$ patients and 30 apparently healthy subjects aged 20-40 years of both sexes were recruited in this study. Patients were diagnosed by psychiatrist following DSM$\mathrm{V}$ criteria. Thirty (30) patients received music therapy (Raga Bhairavi based Rabindra Sangeet) for 3 months and designated as music group. Thirty (30) patients were not given music therapy but followed up after 3 months and designated as non music group. Healthy subjects were not under any music therapy, but studied and at baseline and also followed up after 3 months. Both the patient groups were under similar medication prescribed by psychiatrist throughout the period of 3 months.

Volume 15 No. 1 June 2020: 39-45 
The cardiac autonomic tone of all groups was assessed both at baseline (day 0) and after 3 months of follow up (day 90) by Poincaré analysis of HRV. From the outpatient Department of Psychiatry, BSMMU, the patients were selected and the controls were selected from hospital staff and the attendant of the patients. The protocol of this study was approved by the Institutional Review Board of BSMMU. Patients were excluded if they had psychiatric Co morbidity other than GAD, history of substance abuse or dependence, had a history of cardiovascular, neurological, or endocrinological illness. After enrollment informed written consent was taken from all subjects. After final acceptance the subjects were advised to follow a set of instruction in the previous night in order to prepare for HRV recording in the next day. These included to take their meal within 9:00 pm, remain stress or anxiety free, have a sound sleep, to avoid any sedative on previous night and on the test day they were requested to take light breakfast without tea and coffee and to report the autonomic nerve function test laboratory between 8:00 am to 10:00 am in the Department of Physiology, BSMMU on the test day. An overall physical examinations, including pulse, BP was done and also anthropometric measurements were taken. The subject was given rest for 15-20 minutes in a temperature and sound controlled laboratory environment. In supine position ECG was then recorded in lead II for 6 minutes by a data acquisition device Power Lab 8/35 (AD instrument, Australia). Then poincaré analysis of HRV data was done by the Lab chart software which yielded auto generated report. During this period of data recording he/she was not allowed to talk, eat or drink, to perform physical or mental activity, even sleep. After the baseline data were recorded instruction about the music intervention was given to GAD patients of music group. The patient was previously advised to come with a mobile phone that is capable of playing mp3 music. Then pre selected Raga Bhairavi based Rabindra Sangeet (Tegor's song) were uploaded in his/her mobile. Then, with the help of earphone he/she was advised to listen to the songs in a comfortable position every day morning or any preferable time for half an hour for three consecutive months. By communicating with the attendant of the patient 2/3 times per week via telephone calls better compliance of the therapy was obtained. GAD patient without music therapy and healthy control was advised to come for follow up assessment after 3 months in the same department. For maximum compliance each patient was adequately trained by the researcher before releasing for home practice. Data were expressed as Mean \pm SD. Statistical analysis was done using SPSS version 16. One-way ANOVA followed by Bonferroni's post hoc test and paired sample " $\mathrm{t}$ " test were done for statistical analysis. $p$ value of $<0.05$ was considered as statistically significant.

\section{Results}

At the end of three months, the data of 2 patients of music group and 4 patients of non music group were discarded due to a technical error. Final data of 28 patients of music, 26 patients of non music and 30 healthy subjects at the end of 3 months were used in the analysis. In the present study, all GAD patients were similar to healthy control by age, sex and BMI (Table I). At baseline, mean values of pulse rate, SBP, DBP were found significantly $(\mathrm{p}<.001)$ higher, whereas SD1, SD2 and SD1/SD2 were significantly $(\mathrm{p} \leq 0.01)$ lower in GAD patients than those of normal healthy controls. In addition, all these parameters were almost same in two groups of patients before intervention with music or follow up (Table II). After three months of music therapy, pulse rate, SBP and DBP significantly $(\mathrm{p}<0.01)$ decreased but SD1, SD2 and SD1/SD2 significantly $(\mathrm{p}<$ 0.001 ) increased in GAD patients (Table III), whereas after three months of follow up, the GAD patients not under music therapy, did not show significant changes in these parameters except SD1 when compared to their baseline values. Again, post intervention values of SBP, DBP and SD2 showed lower values in music group compared to their non music counterparts. Furthermore, SD1, SD1/SD2, SBP in music group GAD patients reached close to control value after 3 months (Table IV). 
Table I: Age, sex and BMI of different groups of GAD (N=84)

\begin{tabular}{lccc}
\hline Parameters & $\begin{array}{c}\text { Music } \\
(\mathrm{n}=28)\end{array}$ & $\begin{array}{c}\text { Non M3usic } \\
(\mathrm{n}=26)\end{array}$ & $\begin{array}{c}\text { Control } \\
(\mathrm{n}=30)\end{array}$ \\
\hline Age(years) & $29.79 \pm 5.78$ & $31.69 \pm 6.66$ & $31.1 \pm 3.69$ \\
& $(20-40)$ & $(20-40)$ & $(23-39)$ \\
BMI $(\mathrm{kg} / \mathrm{m} 2)$ & $22.86 \pm 1.52$ & $21.87 \pm 1.96$ & $22.07 \pm 2.12$ \\
& $(18.82-24.77)$ & $(18.59-24.9)$ & $(18.54-24.80)$ \\
Male no (\%) & $14(50 \%)$ & $12(46.15 \%)$ & $15(50 \%)$ \\
Female no (\%) & $14(50 \%)$ & $14(53.85 \%)$ & $15(50 \%)$ \\
\hline
\end{tabular}

Data were expressed as Mean \pm SD. Statistical analysis were done by One-way ANOVA followed by Bonferroni's Post Hoc test; BMI= Body Mass Index; $\mathrm{N}=$ Total number of subjects; $\mathrm{n}=$ number of subjects in each group.

Table II: Baseline values of HRV measures and pulse rate, SBP and DBP in different groups of GAD $(\mathrm{N}=84)$

\begin{tabular}{lccc}
\hline Parameters & $\begin{array}{c}\text { Music } \\
(\mathrm{n}=28)\end{array}$ & $\begin{array}{c}\text { Non music } \\
(\mathrm{n}=26)\end{array}$ & $\begin{array}{c}\text { Control } \\
(\mathrm{n}=30)\end{array}$ \\
\hline SD1 & $14.82 \pm 7.48^{\cdots}$ & $13.81 \pm 8.87^{\cdots}$ & $29.93 \pm 11.88$ \\
& $(4.41-32.76)$ & $(2.21-35.65)$ & $(13.11-56.02)$ \\
SD2 & $42.16 \pm 16.99^{\cdots}$ & $38.21 \pm 17.82^{\cdots}$ & $57.65 \pm 17.47$ \\
& $(16.01-85.75)$ & $(10.65-85.75)$ & $(33.07-101.40)$ \\
SD1/SD2 & $0.35 \pm 0.1^{\cdots}$ & $0.35 \pm 0.19^{\cdots}$ & $0.52 \pm 0.13$ \\
& $(0.22-0.88)$ & $(0.17-1.00)$ & $(0.32-0.84)$ \\
Pulse (beats/min) & $87.11 \pm 11.51^{\cdots}$ & $87.23 \pm 13.94^{\cdots}$ & $73.87 \pm 5.6$ \\
& $(61-110)$ & $(69-125)$ & $(60-90)$ \\
SBP(mm of Hg) & $126.79 \pm 5.13^{\cdots}$ & $122.69 \pm 7.10^{\cdots \cdots}$ & $110.83 \pm 8.10$ \\
& $(110-135)$ & $(110-130)$ & $(100-130)$ \\
DBP(mm of Hg) & $84.11 \pm 5.78^{\cdots}$ & $83.58 \pm 4.12 \cdots$ & $72.00 \pm 5.96$ \\
& $(70-90)$ & $(75-90)$ & $(60-85)$ \\
\hline
\end{tabular}

Data were expressed as Mean $\pm \mathrm{SD}$. Values in parentheses indicate ranges. Statistical analysis were done by One-way ANOVA (among groups) followed by Bonferroni's Post Hoc test (between groups); SBP=Systolic Blood Pressure; DBP=Diastolic Blood Pressure; SD1- Standard deviation of short term RR interval variability; SD2- Standard deviation of Long term RR interval variability; SD1/SD2 ratio-Ratio of short term and long term $\mathrm{RR}$ interval variability $\mathrm{N}=$ number of subjects; $n=$ number of subjects in each group. ${ }^{\cdots}{ }^{=}$music GAD baseline and non music GAD baseline vs. Control baseline. 
Table III: Pre and post intervention/follow up values of HRV measures and pulse rate, SBP and DBP in different groups of GAD $(\mathrm{N}=84)$

\begin{tabular}{|c|c|c|c|c|}
\hline \multirow[t]{2}{*}{ Parameters } & \multicolumn{2}{|c|}{ Music $(n=28)$} & \multicolumn{2}{|c|}{ Non music $(n=26)$} \\
\hline & Pre & Post & Pre & Post \\
\hline \multirow[t]{2}{*}{ SD1 } & $14.82 \pm 7.48$ & $25.19 \pm 16.79 * *$ & $13.81 \pm 8.87$ & $18.43 \pm 11.30$ * \\
\hline & $(4.41-32.76)$ & $(4.8-75.63)$ & $(2.21-35.65)$ & $(2.78-42.85)$ \\
\hline \multirow[t]{2}{*}{$\mathrm{SD} 2$} & $42.16 \pm 16.99$ & $52.41 \pm 24.05^{*}$ & $38.21 \pm 17.82$ & $38.25 \pm 17.92$ \\
\hline & $(16.01-85.75)$ & $(21-144.50)$ & $(10.65-85.75)$ & $(10.65-74.29)$ \\
\hline \multirow[t]{2}{*}{$\mathrm{SD} 1 / \mathrm{SD} 2$} & $0.35 \pm 0.1$ & $0.46 \pm 0.17 * * *$ & $0.35 \pm 0.19$ & $0.42 \pm 0.16$ \\
\hline & $(0.22-0.88)$ & $(0.17-1.00)$ & $(0.17-1.00)$ & $(0.09-0.7)$ \\
\hline Pulse rate & $87.11 \pm 10.51$ & $79.36 \pm 9.63^{* *}$ & $87.23 \pm 13.94$ & $85.19 \pm 10.93$ \\
\hline (beats/min) & $(61-110)$ & $(56-100)$ & $(61-110)$ & $(66-125)$ \\
\hline \multirow[t]{2}{*}{$\mathrm{SBP}(\mathrm{mm}$ of $\mathrm{Hg})$} & $126.79 \pm 5.13$ & $114.46 \pm 7.11^{* * *}$ & $122.69 \pm 7.10$ & $119.81 \pm 6.24$ \\
\hline & $(110-135)$ & $(100-125)$ & $(110-130)$ & $(110-130)$ \\
\hline \multirow[t]{2}{*}{$\mathrm{DBP}(\mathrm{mm}$ of $\mathrm{Hg})$} & $84.11 \pm 5.78$ & $79.82 \pm 5.18^{* *}$ & $83.58 \pm 4.12$ & $83.35 \pm 3.77$ \\
\hline & $(70-90)$ & $(70-85)$ & $(75-90)$ & $(75-92)$ \\
\hline
\end{tabular}

Data were expressed as Mean \pm SD. Statistical analysis was done by Paired sample t-test; $\mathrm{SBP}=\mathrm{Systolic}$ Blood pressure; DBP= Diastolic Blood Pressure; SD1- Standard deviation of short term RR interval variability; SD2Standard deviation of Long term RR interval variability; SD1/SD2 ratio-Ratio of short term and long term RR interval variability $\mathrm{N}=$ Total number of subjects; $\mathrm{n}=$ number of subjects in each group. $* \mathrm{P}<0.05 * * \mathrm{p}<0.01 * * *$ $\mathrm{P}<0.001 .^{*}=$ baseline GAD vs. post music GAD and post non music GAD.

Table IV: Post intervention/follow up values of HRV measures and pulse rate, SBP and DBP in different groups of GAD $(\mathrm{N}=84)$

\begin{tabular}{lccc}
\hline Parameters & Music $(\mathrm{n}=28)$ & Non music $(\mathrm{n}=26)$ & Control $(\mathrm{n}=30)$ \\
\hline SD1 & $25.19 \pm 16.79$ & $18.43 \pm 11.30$ & $28.43 \pm 11.18$ \\
& $(4.8-75.63)$ & $(2.78-42.85)$ & $(12.85-54.73)$ \\
SD2 & $52.41 \pm 24.05^{\$}$ & $38.25 \pm 17.92$ & $53.88 \pm 18.17$ \\
& $(21-144.50)$ & $(10.65-74.29)$ & $(30.97-99.50)$ \\
SD1/SD2 & $0.46 \pm 0.17$ & $0.42 \pm 0.16$ & $0.53 \pm 0.14$ \\
& $(0.22-0.88)$ & $(0.09-0.7)$ & $(0.32-0.84)$ \\
Pulse rate(beats/min) & $79.36 \pm 9.63^{¥}$ & $85.19 \pm 10.10$ & $72.53 \pm 7.48$ \\
& $(56-100)$ & $(66-125)$ & $(62-90)$ \\
SBP(mm of Hg) & $114.46 \pm 7.11^{\$}$ & $119.81 \pm 6.24$ & $112.67 \pm 6.79$ \\
& $(100-125)$ & $(110-130)$ & $(100-125)$ \\
DBP(mm of Hg & $79.82 \pm 5.18^{\$}$ ¥¥¥ & $83.35 \pm 3.77$ & $73.00 \pm 5.66$ \\
& $(70-85)$ & $(75-92)$ & $(60-85)$ \\
\hline
\end{tabular}

Data were expressed as Mean \pm SD. Statistical analysis was done by One-way ANOVA (among groups) followed by Bonferroni's Post Hoc test (between groups); $\mathrm{SBP}=$ Systolic Blood pressure; $\mathrm{DBP}=$ Diastolic Blood Pressure SD1- Standard deviation of short term RR interval variability; SD2- Standard deviation of Long term RR interval variability; SD1/SD2 ratio-Ratio of short term and long term RR interval variability; $N=$ Total number of subjects; $n=$ number of subjects in each group. ${ }^{\$}=$ post music GAD vs. post non music GAD; ${ }^{\$=} \mathrm{P}<0.05$; ${ }^{¥=}$ post music GAD vs. control follow up. ${ }^{¥=} \mathrm{P}<0.05,{ }^{¥}=<0.01,{ }^{¥ ¥}=<0.001$, 
Discussion

In this study, significantly higher resting pulse rate, SBP, DBP and lower SD1, SD2 and SD1/ SD2 in newly diagnosed GAD patients before intervention compared to control suggested cardiac autonomic dysfunction with poor parasympathetic tonic activity in all drug naive GAD patients. These results agree to some investigators those who have observed association of hypertension and increased risk of cardiovascular disease (CVD) with GAD. ${ }^{19-}$

21 Thus these observations of derangement of autonomic tone in the current series of patients also clearly indicate the risk of CVD in these GAD patients. After listening Rag based song for three months the significant decrement of pulse rate, SBP and DBP in patients from their baseline suggested improvement of cardiac autonomic regulation in this group of patients. These findings also agreed to others. ${ }^{22-24}$ Similar changes were also observed by others but in case of stress. ${ }^{25}$ Moreover, SBP in music group of patient were close to healthy subjects which in turn strongly support the good effect of music on autonomic regulation.

Again significant increment in SD1, SD2 and SD1/SD2 after listening music for 3 months demonstrate the effect of music therapy on improvement of parasympathetic and sympathetic tone in GAD patients. This observation is further supported by the results of these parameters in GAD patients without music therapy where no significant change in these parameters was found after 3 months of follow up. In addition, it was apparent that 3 months of music therapy could restore these parameters close to control value.

But several other investigators reported similar effects on SD1/SD2 by using musical instrument to produce auditory stimulus. ${ }^{26-27}$ Previous studies have also found similar findings in case of Rabindra Sangeet on normal healthy individuals. $^{28}$

How music therapy affects the cardiac autonomic nerve function is not still well explained. It has been suggested that physiological relaxation due to music therapy might be responsible for increase in parasympathetic activity and decrease in sympathetic activity. $28-29$

\section{Conclusion}

From the results of the present study, it can be finally concluded that music therapy can effectively improve autonomic dysfunction in GAD and it can be successfully used as an adjunct to medication and thereby protect the patients with generalized anxiety disorder from cardiovascular risk.

\section{Ethical Issue}

The ethical clearance was acquired from the Institutional Review Board of the University. By the $183^{\text {rd }}$ Institutional Review Board, Bangabandhu Sheikh Mujib Medical University approval paper was given and meeting held on 15th June 2019 (No. BSMMU /2019 /6914).

\section{Conflict of Interest}

No conflict of interest was declared by the Authors.

\section{References}

1. American Psychiatric Association. Diagnostic and Statistical manual of mental disorders (DSM-5). $5^{\text {th }}$ ed. Washington DC: APA ; 2013.155-68.

2. Alkhader YK. Generalized anxiety disorder: a review. IJMDC. 2018; 2: 65-9.

3. Islam MR, Ahmed MU, Islam MS, Sayeed MS, Sadia F, Chowdhury ZS, Nahar Z, Hasnat A. Comparative analysis of serum malondialdehyde, antioxidant vitamins and immunoglobulin levels in patients suffering from generalized anxiety disorder. Drug Res 2014; 64: 406-11.

4. Kim K, Lee S, Kim JH. Diminished autonomic neurocardiac function in patients with generalized anxiety disorder. Neuro Psychiatr Dis Treat 2016; 12: 3111.

5. Chang HA, Chang CC, Tzeng NS, Kuo TB, Lu RB, Huang SY. Generalized anxiety disorder, comorbid major depression and heart rate variability: a casecontrol study in taiwan. Psychiatry Invest 2013; 10: 326.

Volume 15 No. 1 June 2020: 39-45 
6. Tully PJ, Cosh SM, Baune BT. A review of the affects of worry and generalized anxiety disorder upon cardiovascular health and coronary heart disease. Psychol Health Med. 2013; 18: 627-44.

7. Chalmers JA, Quintana DS, Abbott MJ, Kemp AH. Anxiety disorders are associated with reduced heart rate variability: a meta-analysis. Front Psychiatry. 2014; 5: 80.

8. Acharya UR, Joseph KP, Kannathal N, Lim CM, Suri JS. Heart rate variability: a review. Med Bio Eng Comput. 2006; 44: 1031-51.

9. Massaro S, Pecchia L. Heart rate variability (HRV) analysis : a methodology for organizational neuroscience. Organ Res Methods 2016; 22:1-40.

10. Hsu C-H, Tsai M-A, Huang G-S, L T-C, Chen K-P, Ho S-T, Shyu LY, Li C-Y. Poincare plot index of heart rate variability detect dynamic autonomic modulation during general anesthesia induction. Acta Anaesthesiol Taiwan 2012; 50:12-18.

11. Voss A, Schulz S, Schroeder R, Baumert M, Caminal P. Methods derived from nonlinear dynamics for analyzing heart rate variability. Philos Trans A Math Phys Eng Sci 2009; 367: 277-96.

12. Hoshi RA, Pastre CM, Vanderlei LC, Godoy MF. Poincaré plot indexes of heart rate variability: relationships with other nonlinear variables. Auton Neurosci 2013; 177: 271-4.

13. Kahloul M, Mhamdi S, Nakhli MS, Sfeyhi AN, Azzaza M, Chaouch A, Naija W. Effects of music therapy under general anesthesia in patients undergoing abdominal surgery. Libyan J Med 2017; 12: 1260886.

14. Sharma M, Rajnee and Mathur KC. Effects of music therapy on clinical and biochemical parameters of metabolic syndrome. J Bangladesh Soc Physiol 2011; 6: 108-15.

15. Gold C, Heldal TO, Dahle T, Wigram T. Music therapy for schizophrenia or schizophrenia like illnesses. Cochrane Database Sys Rev 2005; CD 004025.

16. Roque AL, Valenti VE, Guida HL, Campos MF, Knap A, Vanderlei LCM, Ferreira LL, Ferreira C, Abreu LC. The effects of auditory stimulation with music on heart rate variability in healthy women. Clinics 2013; 68: 960-7.

17. Islam SR, Ferdousi S. Music therapy on nonlinear assessment of cardiac autonomic function in patients with major depressive disorder. J Bangladesh Soc Physiol 2019;14: 7-13.

Volume 15 No. 1 June 2020: 39-45
18. Gutiérrez EO, Camarena VA. Music therapy in generalized anxiety disorder. Arts Physio 2015; 44: 1924.

19. Makovac E, Meeten F, Watson DR, Herman A, Garfinkel SN, Critchley HD, Ottaviani C. Alterations in amygdala-prefrontal functional connectivity account for excessive worry and autonomic dysregulation in generalized anxiety disorder. Biol Psychiatry 2016; 80: 786-95.

20. Hoehn-Saric R, McLeod DR, Funderburk F, Kowalski P. Somatic symptoms and physiologic responses in generalized anxiety disorder and panic disorder: An ambulatory monitor study. Arc Gen Psychiat 2004; 61: 913-21.

21. Barger SD, Sydeman SJ. Does generalized anxiety disorder predict coronary heart disease risk factors independently of major depressive disorder? J Affect Disord 2005; 88: 87-91.

22. Chennafi M, Khan MA, Li G, Lian Y, Wang G. Study of music effect on mental stress relief based on heart rate variability. In2018 IEEE Asia Pacific Conference on Circuits and Systems. .APCCAS 2018:131-4.

23. Lee KS, Jeong HC, Yim JE, Jeon MY. Effects of music therapy on the cardiovascular and autonomic nervous system in stress-induced university students: a randomized controlled trial. J Altern Complem Med 2016; 22: 59-65.

24. Sharma M, Mathur KC. Effects of music therapy on clinical and biochemical parameters of metabolic syndrome. J Bangladesh Soc Physiol 2011; 6:108-15.

25. Latha R, Srikanth S, Sairaman H, Dity NR. Effect of music on heart rate variability and stress in medical students. Int J Clin Exp Physiology 2014;1: 131-4

26. Roque AL, Valenti VE, Guida HL, Campos MF, Knap A, Vanderlei LCM, Ferreira LL, Ferreira C, Abreu LC. The effects of auditory stimulation with music on heart rate variability in healthy women. Clinics 2013; 68: 960-7.

27. Roy B, Choudhury R, Pandey A, Bandopadhyay S, Sararangi S, Ghatak SK. Effect of rotating acoustic stimulus on heart rate variability in healthy adults. Open Neurol J 2012; 6: 71-7.

28. Mukherjee S, Palit SK, Banerjee S, Bhattacharya DK. A comparative study on three different types of music based on same indian raga and their effects on human autonomic nervous systems, in Chaos, Complexity and Leadership, Erçetin aa, Banerjee S, editors. Cham: Springer International Publishing; 2013, 243-54.

29. Trappe HJ. The effects of music on the cardiovascular system and cardiovascular health. Heart 2010; 96: 1868-71. 